\title{
TREC-IN: gene knock-in genetic tool for genomes cloned in yeast
}

\author{
Suchismita Chandran', Vladimir N Noskov', Thomas H Segall-Shapiro', Li Ma' ${ }^{1}$ Caitlin Whiteis ${ }^{1}$, Carole Lartigue ${ }^{2,3}$,
} Joerg Jores ${ }^{4}$, Sanjay Vashee ${ }^{1}$ and Ray-Yuan Chuang ${ }^{1 *}$

\begin{abstract}
Background: With the development of several new technologies using synthetic biology, it is possible to engineer genetically intractable organisms including Mycoplasma mycoides subspecies capri (Mmc), by cloning the intact bacterial genome in yeast, using the host yeast's genetic tools to modify the cloned genome, and subsequently transplanting the modified genome into a recipient cell to obtain mutant cells encoded by the modified genome. The recently described tandem repeat coupled with endonuclease cleavage (TREC) method has been successfully used to generate seamless deletions and point mutations in the mycoplasma genome using the yeast DNA repair machinery. But, attempts to knock-in genes in some cases have encountered a high background of transformation due to maintenance of unwanted circularization of the transforming DNA, which contains possible autonomously replicating sequence (ARS) activity. To overcome this issue, we incorporated a split marker system into the TREC method, enabling seamless gene knock-in with high efficiency. The modified method is called TREC-assisted gene knock-in (TREC-IN). Since a gene to be knocked-in is delivered by a truncated non-functional marker, the background caused by an incomplete integration is essentially eliminated.

Results: In this paper, we demonstrate applications of the TREC-IN method in gene complementation and genome minimization studies in Mmc. In the first example, the Mmc dnaA gene was seamlessly replaced by an orthologous gene, which shares a high degree of identity at the nucleotide level with the original Mmc gene, with high efficiency and low background. In the minimization example, we replaced an essential gene back into the genome that was present in the middle of a cluster of non-essential genes, while deleting the non-essential gene cluster, again with low backgrounds of transformation and high efficiency.

Conclusion: Although we have demonstrated the feasibility of TREC-IN in gene complementation and genome minimization studies in Mmc, the applicability of TREC-IN ranges widely. This method proves to be a valuable genetic tool that can be extended for genomic engineering in other genetically intractable organisms, where it may be implemented in elucidating specific metabolic pathways and in rationale vaccine design.
\end{abstract}

Keywords: Mycoplasma, TREC, Gene knock-in, Genomes, Yeast, Autonomously replicating sequence, Genome minimization

\section{Background}

Mycoplasmas are the simplest and smallest living prokaryotes $(0.1 \mu \mathrm{m})$, and although phylogenetically related to Gram-positive bacteria, lack a cell wall [1]. They also have the smallest recorded genomes (0.58 Megabases $(\mathrm{Mb})-1.38 \mathrm{Mb})$ for bacterial species that can replicate autonomously, and have colonized a wide range of hosts

\footnotetext{
* Correspondence: rchuang@jcvi.org

${ }^{1}$ The J. Craig Venter Institute, 9704 Medical Center Drive, Rockville 20850, MD, USA

Full list of author information is available at the end of the article
}

including, humans and animals [2]. However, efforts to manipulate mycoplasma genomes are fraught with difficulties owing to the lack of genetic tools available for these organisms [3]. This has made understanding the biology and elucidating the host-pathogen mechanism for any potential therapeutics, including vaccine development, challenging.

One of the early genetic tools that were developed for understanding mycoplasma biology was the generation of $\mathrm{OriC}$ plasmids that could replicate in mycoplasma cells [4-6]. Although heterologous gene expression and 
targeted gene disruption by single-crossover recombination were demonstrated in Mycoplasma mycoides subspecies capri $(M m c)$ and $M$. capricolum subspecies capricolum $(M c c)$, no recombination events were observed in the closely related $M$. mycoides subspecies mycoides $(\mathrm{Mmm})[7,8]$. In addition, maintaining stable mutants using OriC plasmids turned out to be difficult and laborious $[4,7,8]$. Thus, alternate strategies were designed, including a transposon-based method to generate mutants with low passage numbers that were free of antibiotic-resistance genes [9]. Transposon-based mutagenesis has been prevalently used as a genetic tool in mycoplasmas to generate mutants of interest as well as to define essential genes required for survival [10-12]. Furthermore, a double-crossover homologous recombination method using a suicide plasmid has been described for M. genitalium, albeit at a very low frequency [13-15], but this method did not address seamless deletion and removal of markers [16]. Therefore, to overcome stability and marker recycling issues, we turned to yeast genetics and synthetic biology to extend the genetic toolbox of mycoplasmas. With recent advancements in synthetic genomics including, cloning of the $M m c$ genome in yeast, manipulation of the mycoplasma genome using yeast genetic tools, transplantation of the engineered mycoplasma genome from yeast to a bacterial recipient cell, and creation of the synthetic cell, expression of the engineered genome became possible [17-22]. Mycoplasma genomes including $M$. genitalium (0.6 Mb), M. pneumoniae $(0.8$ $\mathrm{Mb})$, and $M m c(1.1 \mathrm{Mb})$ were first cloned into yeast with the idea of implementing yeast genetics tools to engineer genetically intractable organisms [17-22].

Once cloned in yeast, bacterial genomes can be theoretically manipulated by yeast genetic tools. The URA3 marker/5-FOA counter-selection is a common technique in which the marker can be recycled to create seamless gene deletions, replacements, or gene knock-ins. However, we have previously shown that this conventional two-step method was very inefficient in engineering a mycoplasma genome cloned in yeast due to instability of the genome where high background of 5-FOA resistant colonies resulted from non-specific removal of the URA3 marker [22]. Development of the tandem repeat coupled with endonuclease cleavage (TREC) method has greatly improved the efficiency of seamless gene deletions $[21,22]$. TREC can be also applied in gene knock-in via a single step transformation where the knock-in sequence is placed outside the cassette and immediately next to the repeated sequence (Figure 1). The removal of the cassette leaves the knock-in sequence in the target site seamlessly. Although TREC method is currently the best tool that can seamlessly engineer a genome cloned in yeast $[21,22]$, the process is sometimes inefficient with a high background of transformation, arising possibly due to illegitimate recombination (Figure 1). To overcome this limitation of TREC-mediated gene insertion, we developed a modified method, called TREC assisted gene knock-in (TREC-IN) that significantly improves the efficiency of gene knock-in and vastly reduces screening effort. This method relies on the split marker system whereby the gene is delivered by a non-functional truncated antibiotic resistant gene module $\operatorname{kan} M X$, and a sitespecific gene insertion is selected by functional restoration of the full length $\operatorname{kan} M X$ gene. Here, we demonstrate the feasibility of TREC-IN in the $M m c$ genome using two examples a) replacement of an endogenous gene with an orthologous one, and b) essential gene complementation in a genome reduction study.

\section{Results}

\section{Design of the TREC-IN}

The design of TREC-IN is based on the previous TREC strategy and incorporates a split marker approach with an additional step for a gene knock-in that is mediated by a functional restoration of the kanamycin resistance gene module, $k a n M X$. The procedure involves three steps: first, insertion of a CORE6 cassette to the target locus; second, site-specific gene integration; and third, seamless cassette recycling (Figure 2A). In the first step, the CORE6 cassette, which consists of the $18 \mathrm{bp}$ I-SceI binding site, the I-SceI endonuclease encoding gene under the control of the yeast GAL1 promoter, the KlURA3 gene and a $5^{\prime}$ truncated $\operatorname{kanMX}$ gene component, is introduced to the target site. Similar to the TREC method, two sequences of about $50 \mathrm{bp}$ that are homologous to the target site are added into the CORE6 cassette by PCR on $5^{\prime}$ and $3^{\prime}$ ends of the cassette so that they flank the CORE6 cassette (Figure 2B). Transformation of the CORE6 cassette into yeast and homologous recombination at the target site results in the replacement of the target site by the CORE6 cassette. Transformed yeast colonies are selected for uracil prototrophs, and further analyzed by PCR screening to confirm that the homologous recombination has occurred at the correct target site (Figure 2A). The second step of TREC-IN involves construction and transformation of the knock-in module containing a $3^{\prime}$ truncated kanMX gene component and the knock-in sequence. The kanamycin resistance gene and the knock-in sequence are separated by a repeat sequence of about $50 \mathrm{bp}$ in length, which is identical to upstream sequences of the target site in the CORE6 cassette (Figure 2C). This knockin module is flanked at the $5^{\prime}$ end by a region of the kanamycin resistance gene to allow for homologous recombination at the 3 ' end of the CORE6 cassette. On its $3^{\prime}$ end, the knock-in module is flanked by the same homologous region that is also present on the 3 ' end of the CORE6 cassette to allow for recombination 


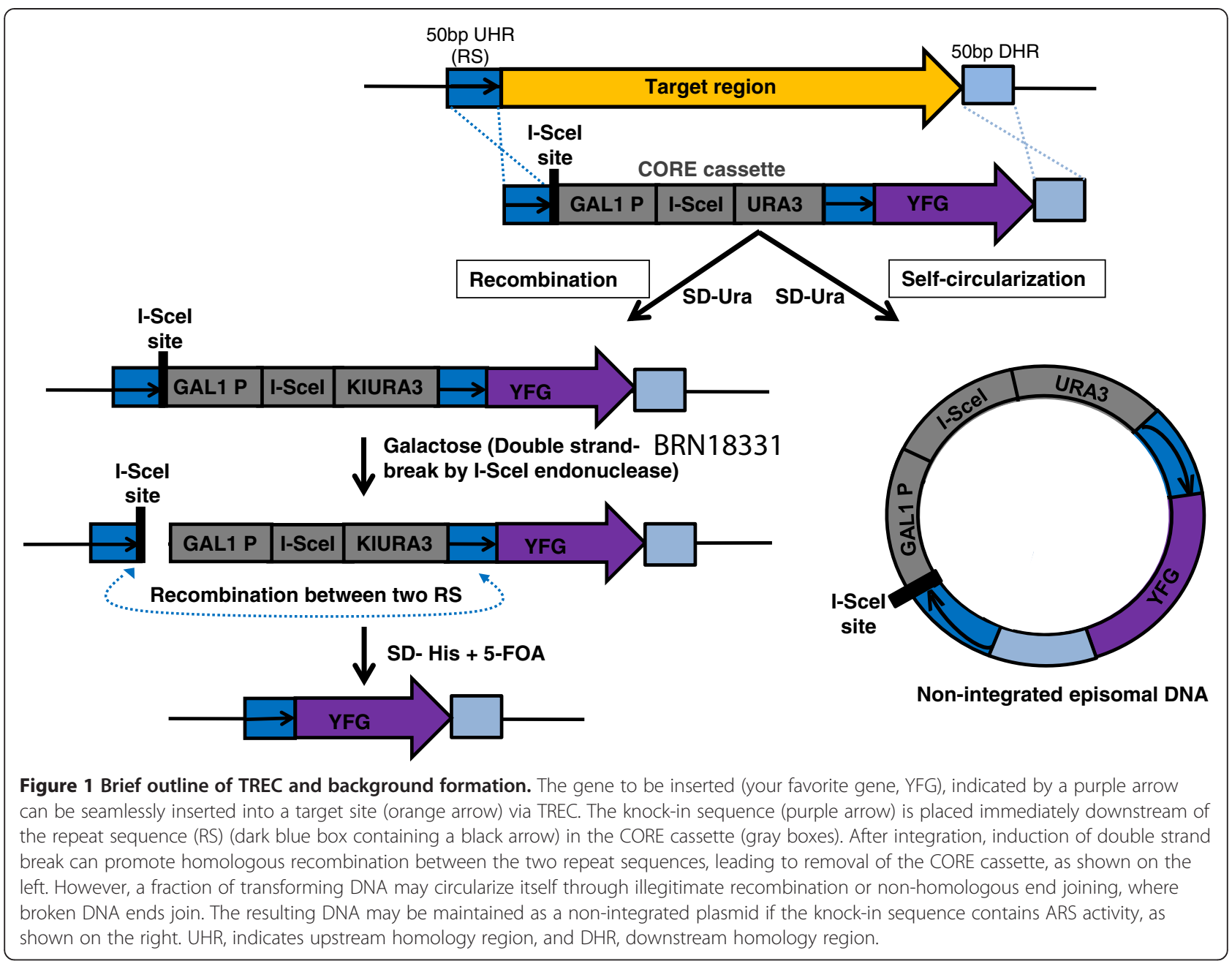

at the target site (Figure 2A). Upon transformation, the knock-in module integrates into the target site, resulting in an insertion containing two repeat sequences encompassing three genes (the I-SceI, KlURA3, and the full length $k a n M X$ module) and the knock-in sequence. Transformed yeast colonies are selected for resistance against the antibiotic geneticin, and then analyzed by PCR screening to confirm correct insertion. In the third step of TREC-IN, the whole cassette flanked by the two repeat sequences is removed via homologous recombination between the two repeat sequences. The efficiency of the recombination is enhanced by the double strand break (DSB) generated by the cleavage of the endonuclease I-SceI at the 18 bp recognition site in the cassette after galactose induction. The removal of the KlURA3, I-SceI, and the $\operatorname{kanMX}$ module counter-selected by 5 -FOA would leave no scar. Only the knock-in sequence remains at the target site. Yeast cells that are resistant to 5-FOA are screened by PCR for the precise insertion of the replacement sequence (Figure 2).
Replacement of the Mcc orthologous dnaA gene in the Mmc genome

To demonstrate precise replacement of an orthologous gene in the $M m c$ genome, TREC-IN was applied to replace the $M m c$ dnaA gene, which is essential for chromosomal replication and viability $[5,19]$, with the orthologous dnaA gene from Mcc. The Mmc (accession no. AY277700) and $M c c$ dnaA genes (accession no. D90426) share 95\% sequence identity at both the nucleotide and protein levels (analyzed using BLAST). As described in Methods, the first step of TREC-IN resulted in the precise replacement of the endogenous $d n a A$ gene by the CORE6 cassette (Figure $3 \mathrm{~A}$, step 1). The promoter and 3' region of the $M m c d n a A$ gene were left unaltered. In the second step of TREC-IN, the orthologous Mcc dnaA gene was integrated downstream of the CORE6 cassette under geneticin selection (Figure 3A, step 2). In the third step, DSB at the I-SceI site promoted homologous recombination between the two repeat sequences, followed by precise and seamless insertion of the $M c c d n a A$ gene in the 


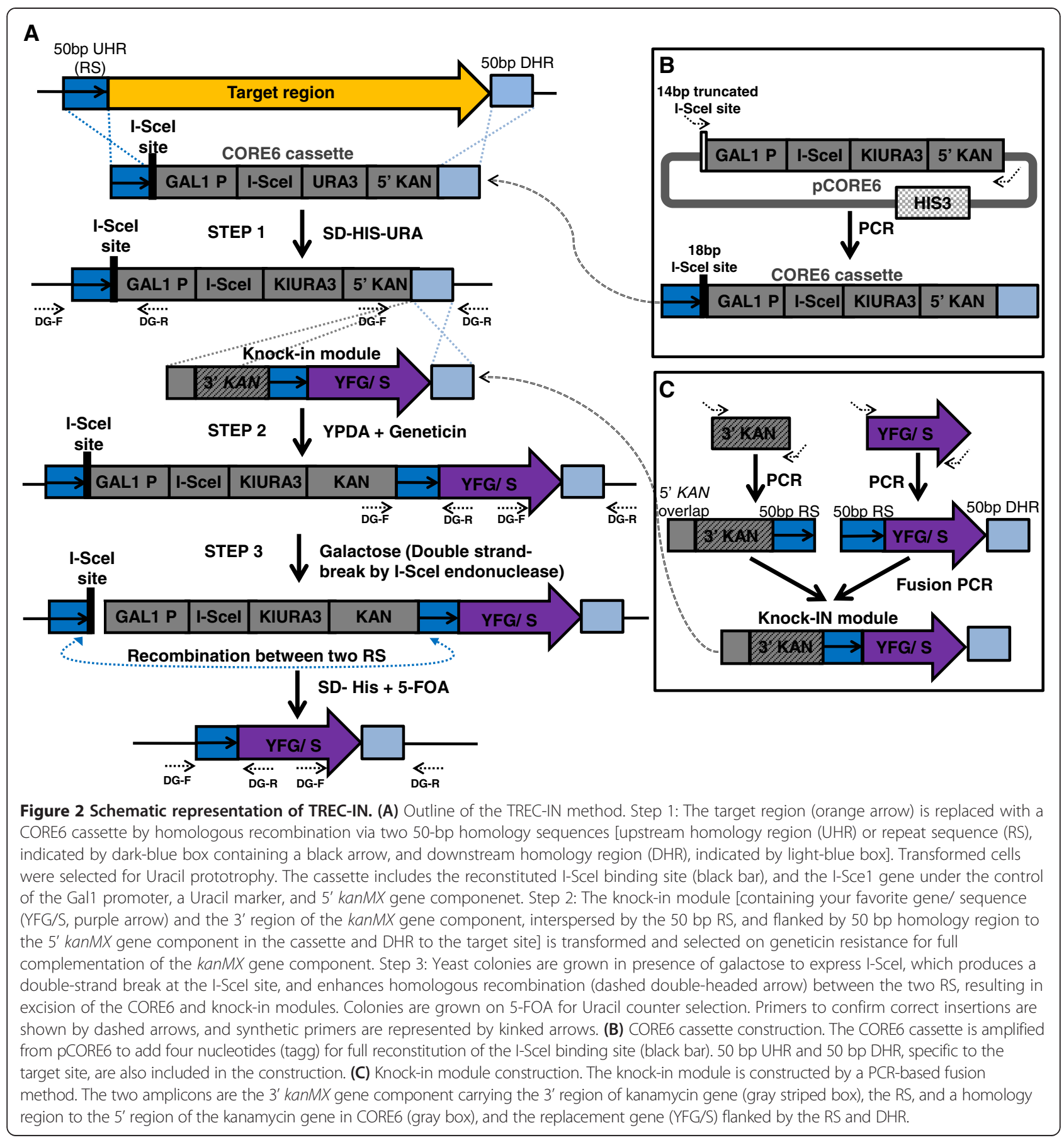

$M m c$ genome (Figure 3A, step 3). Each step of the deletion and replacement procedure was evaluated by PCR screening to confirm the correct insertions and junctions (Figure 3B).

Upon transformation, seven colonies were screened by PCR and all the colonies were found to be positive for CORE6 replacement [Figure 3B (a)]. In the second step, we PCR-screened 36 geneticin-resistant colonies for multiple junctions and found that 33/36 (>91\%) of the colonies were positive for the precise insertion of the cassette at the targeted locus [Figure 3B (b)]. In the third step, I-SceI-mediated DSB resulted in 27/36 (75\%) of the colonies showing precise removal of the cassette, resulting in a clean insertion [Figure 3B (c)]. Of note, PCRscreening [Figure 3B (b) and 3B (c)] indicated that while majority of the colonies obtained resulted in seamless replacement of the knock-in gene, the remaining 9\% and $25 \%$ colonies respectively, were positive for only one or 


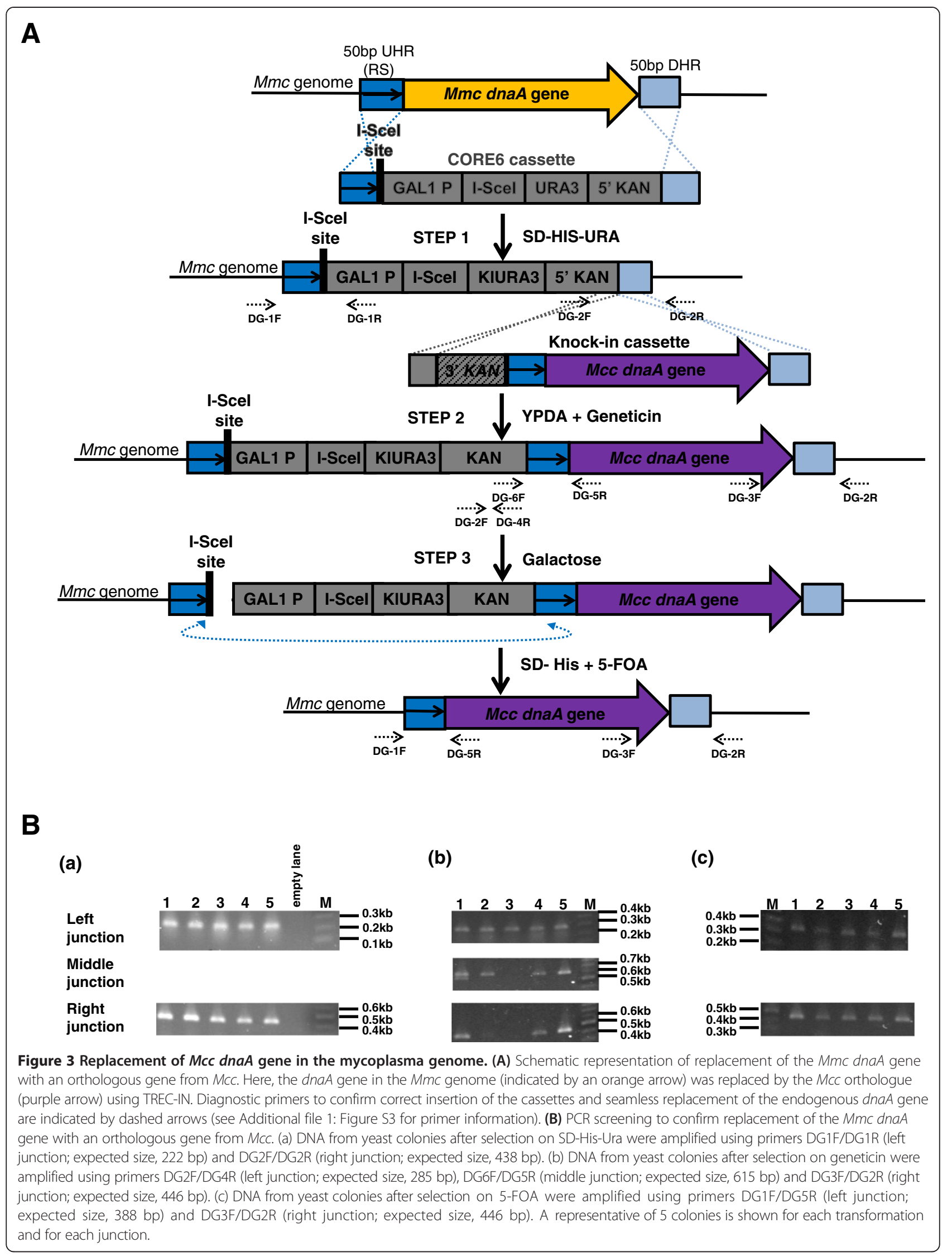


two of the junctions tested, suggesting non-specific recombination. Thus, TREC-IN proves to be a valuable genetic tool to overcome background issues and facilitate gene knock-in experiments with increased efficiency.

Both, the dnaA gene-deleted and dnaA gene-complemented $M m c$ genomes were transplanted to generate the mutant $M m c$ strains, as described previously [21]. As expected, genome transplantation of the $d n a A$ gene-deleted genome resulted in non-viability. However, replacement of the orthologous Mcc dnaA gene resulted in a viable cell. The resulting colonies were of similar size to those of the control wild-type $M m c$ colonies (data not shown). Genomic DNA from the $d n a A$-replaced $M m c$ cells was isolated and analyzed by sequencing to confirm the precise and scarless insertion of the $M c c$ dnaA gene.

\section{Application of TREC-IN for genome reduction in the $\mathrm{Mmc}$ genome}

Global transposon random mutagenesis has been widely used to identify non-essential genes in minimal genome studies in prokaryotes [10-12]. Therefore, using transposons, we generated a high-resolution map of non-essential gene candidates on a synthetic $M m c$ genome (unpublished data). To carry out a top-down genome reduction strategy, consecutive non-essential genes were grouped into multigene deletion targets and were labeled non-essential gene clusters (NEGC). In some cases, several NEGCs were interspersed by single or a few Tn5-defined essential genes. To achieve genome reduction more efficiently, the TRECIN approach was tested to remove multiple NEGCs simultaneously, and then add back the essential genes to the genome that were interspersed between them. To demonstrate this application, we chose a $16 \mathrm{~kb}$ region of the synthetic $M m c$ genome (Mmc Syn1) covering two NEGCs consisting of 10 genes, separated by a Tn5-denfined essential gene $(s s r A)$ for deletion (Figure $4 \mathrm{~A}$ and Additional file 1: Figure S2). In the first step of TREC-IN, the integration of the CORE6 cassette at the target site resulted in the deletion of the two NEGCs (Mmc Syn1 0152-0157 and Mmc Syn1 0159-0162) along with the intervening essential gene $s s r A$ (Mmc Syn1 0158) from the $M m c$ Syn1 genome. In the second step of TREC-IN, transformation of the knock-in module resulted in the precise insertion of the $M m c s s r A$ gene back into the synthetic $M m c$ genome. The precise cluster deletion followed by insertion of the $s s r A$ gene was verified by PCR screening (Figure $4 \mathrm{~B}$ ). The phenotypes of both cluster-deleted and ssrA gene-complemented $M m c$ strains were determined by genome transplantation. We found that the whole $16 \mathrm{~kb}$ deletion comprising 11 genes resulted in a nonfunctional genome as observed by the lack of viable cells. However, cis-complementation of the ssr $A$ gene rescued the lethal phenotype. Transplantation colonies from the ssrA complemented synthetic $M m c$ genome were viable, and showed similar colony size to those of the control synthetic $M m c$ cells (data not shown). Genomic DNA from the $s s r A$ complemented $M m c$ cells was isolated and analyzed. Sequencing of the complemented ssr $A$ region in the isolated modified synthetic $M m c$ genome confirmed the precise and seamless insertion of the essential $M m c$ ssr $A$ gene and deletion of the two NEGCs.

\section{Discussion}

Mycoplasmas infect a wide range of hosts, including humans and animals, and in some cases, even contribute towards economic havoc [1,2]. Therefore, developing better genetic tools to study and contain these pathogens has become a priority. $M m c$, with its relatively small genome, and ease of manipulation $[20,21]$ is not only being probed as a model to study pathogenesis, but also as a model organism to test the concept of a minimal cell, where essential genes and functions are being determined. Additionally, $M m c$ is also being modeled as a platform to develop tools towards vaccine development that can be applied to other mycoplasma species. However, the existing genetic tool box makes it difficult to study this bacterial species.

With recent advancements in synthetic genomics, it is now possible to engineer the $M m c$ genome using yeast genetic tools, including TREC [17-22]. Development of TREC is based on a modified yeast system where generation of seamless deletions [22] and point mutations (unpublished data) in the mycoplasma genome is now made possible by using the yeast DNA repair machinery. In principle, the TREC method can be employed to insert genes of interest into the $M m c$ genome. However, several attempts to knock-in an $M m c$ gene into the $M m c$ genome were inefficient with a high background of transformation. Since yeast ARSs are A-T rich, and the mycoplasma genome is relatively A-T rich, it is reasonable to speculate that gene knock-ins containing A-T rich sequences of mycoplasma genomes likely contain ARS activity [17]. Akada and colleagues reported that a gene containing an ARS performs inefficient chromosomal integration [23]. Thus, a portion of the transforming DNA can circularize through illegitimate recombination or NHEJ [24], and be maintained as a non-integrated free plasmid in the yeast cell (unpublished results, CL). To circumvent this problem, we developed TREC-IN, which can efficiently produce gene knock-ins without leaving any scars. Since the TREC-IN method encompasses elements of the TREC method and a split marker system for seamless replacement of nucleotide sequences at any given location on the genome, background issues arising from unwanted ARS activity and A-T rich content are greatly reduced. In the example of $d n a A$ gene replacement (Figure 2) TREC-IN was employed to replace the A-T rich $M m c$ gene with an orthologous gene from $M c c$. However in this case, direct 


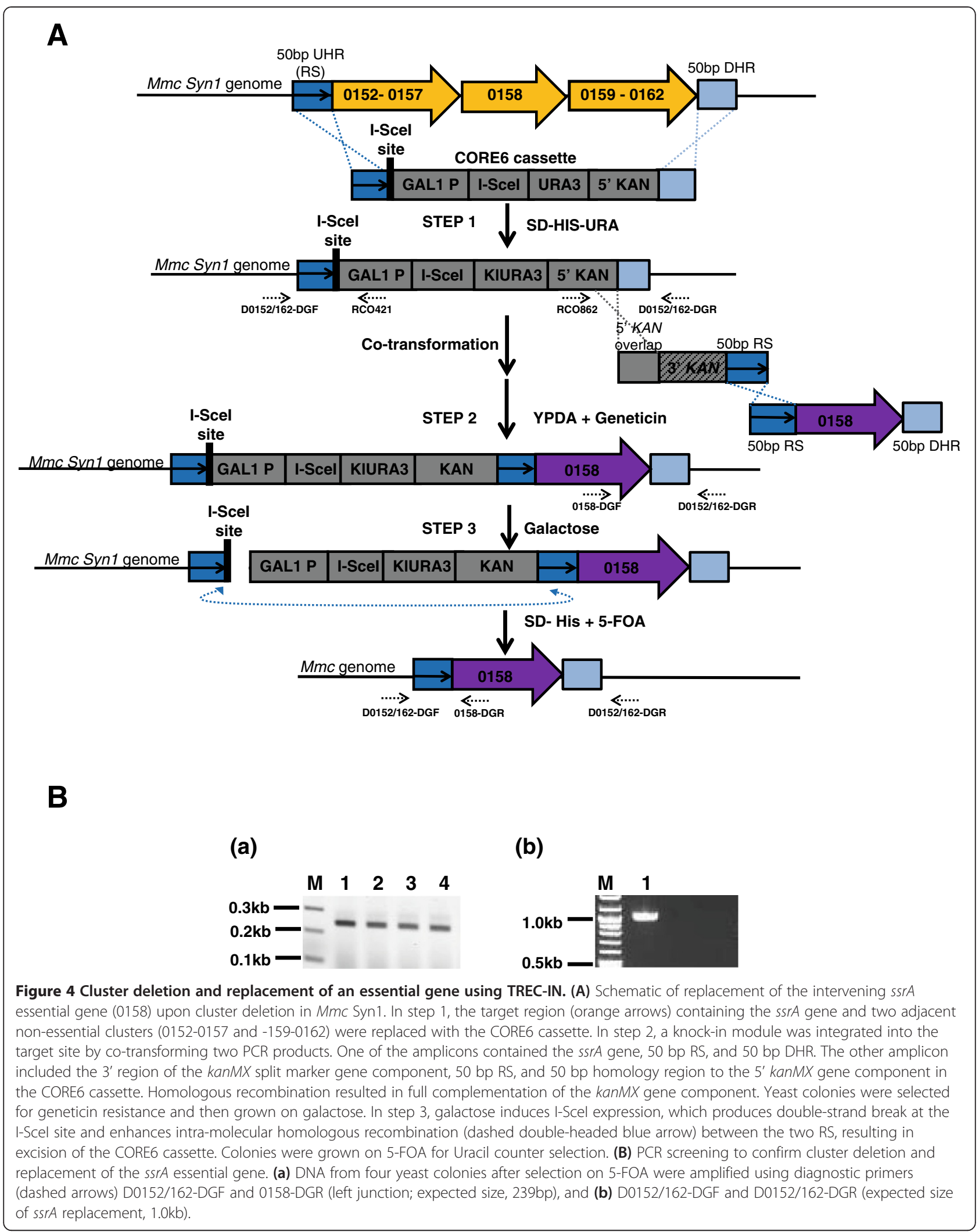


comparison with TREC was not possible because the orthologous genes share a high degree of homology (95\%) and the TREC design would not be able to resolve partial recombination occurring between the two genes, as expected. Therefore by using TREC-IN, efficiency of replacement is vastly improved with frequencies of obtaining a positive clone nearing 75\% (see Results), thus circumventing the cumbersome screening process of TREC which would be labor and time intensive. Of note, comparing efficiencies between TREC and TREC-IN proves to be complicated as it varies on a case-case basis where A-T content and secondary structure has to be taken into consideration.

Furthermore, TREC-IN can also been extended to delete genes with possible ARS-like activity from the $M m c$ genome, which are very difficult to achieve by the TREC method. For example, we made several attempts to delete the glycerol facilitator $(g l p F)$ gene from the $M m c$ genome using TREC (unpublished results). We found transformations yielded increased levels of background colonies growing on selective media, without the correct replacement. Yet, when the TREC-IN strategy was applied to delete the $M m c \operatorname{glpF}$ gene, all colonies obtained contained the precise and seamless deletion of the $g l p F$ gene (manuscript in preparation, SC, LM, CL, JJ, RC, SV). In contrast to the TREC method, no background colonies were observed. While TREC-IN depends upon integration of the CORE cassette to the target site, there is some flexibility in choosing the integration sites. Depending on the case, the design can be modified to target sequences that may reside in upstream or downstream adjacent genes if the original target sites prove to be difficult. The adjacent genes can then be restored by including the deleted sequence in the knock-in fragment. The $g l p F$ deletion described above provides such an example. In this case, the design for the downstream homologous region (Figure 2) was modified to include part of the neighboring $g l p K$ gene, in order to bypass a specificity issue at the 3' end of the $g l p F$ gene. The missing $M m c$ glpK region was complemented in the second step of the TREC-IN method.

TREC has been employed to delete target sites of greater than $70 \mathrm{~kb}$ seamlessly in $\mathrm{Mmc}$ (unpublished results), and since TREC-IN utilizes elements of the TREC, it can be speculated that TREC-IN can also be used to seamlessly delete similarly large nucleotide tracts. Although analysis of knock-in sequences larger than $3 \mathrm{~kb}$ has not been carried out, it is theoretically possible that TREC-IN would be able to handle larger fragments, possibly with lower efficiency in trying to complete homologous recombination. In summary, TREC-IN proved to be useful in modifying regions of the genome that tend to be difficult to engineer either due to high A-T content or ARS activity, where TREC or other conventional yeast genetic tools maybe limiting.

\section{Conclusion}

The TREC-IN method proves to be a powerful genetic tool for manipulating mycoplasma genome. In addition to finding applications in our top-down genome minimization of $M m c$ (Figure 4), this method can be employed to explore homologous complementation studies in other related organisms, including $M$. leachii, and $M$. putrefaciens efficiently without the cumbersome screening process that would be required by TREC alone. By using TREC-IN to manipulate metabolic pathways, pathogenic and virulence factors may be studied with relative ease; thereby facilitating better vaccine design against some of the economically devastating livestock diseases such as contagious bovine pleuropneumonia caused by mycoplasmas [25]. In our studies of mycoplasma biology, TREC and TREC-IN dramatically increased our ability to manipulate the genomes of genetically intractable bacteria. As synthetic genomics techniques are extended to other bacteria that are difficult to manipulate genetically, TREC and TREC-IN will become even more valuable as tools for engineering bacterial genomes cloned as yeast centromeric plasmids.

\section{Methods}

\section{Yeast strain and media}

The yeast Saccharomyces cerevisiae, strain VL6-48 (MATahis3$\triangle 200$ trp1- $\Delta 1$ KlURA3- $\Delta 1$ lys2 ade2-101 met14) containing either the $1.08 \mathrm{Mb}$ genome of Mycoplasma mycoides subspecies capri $(\mathrm{Mmc})$ with a yeast centromeric plasmid (YCP) [21], or the synthetic $M m c$ genome (Mmc Syn1) [19] were employed. Yeast cells were grown and maintained in either the synthetic minimal medium containing dextrose (SD) [21], or the standard rich medium containing glucose (YPD) or galactose (YPG) [22]. SD medium was supplemented with 5-fluoroorotic acid (5-FOA), for KIURA3 counter-selection [21,26].

\section{Preparation of mutagenesis cassettes \\ A. Construction of pCORE6 plasmid}

The pCORE6 plasmid (GenBank accession number KP282615) was constructed by cloning the $5^{\prime}$ region of the kanamycin resistance gene along with its promoter (5' KanMX, 1-859 bp) into the previously constructed pCORE3 plasmid (unpublished) at the EcoR I site (Additional file 1: Figure S1). More precisely, 5' $\mathrm{kanMX}$ was amplified from the previously described pFA6a-KanMX plasmid [27] using primers, RCO858 (CAGGAATTC GACATGGAGGCCCAGAATAC) and RCO859 (ATC GAATTCGGCCAGCCATTACGCTCGT), containing the EcoR I restriction site (GAATTC) at each extremity. The pCORE3 plasmid, which includes a 14 bp incomplete I-SceI binding site (white box), a Gal1 promoter, an I-SceI gene, and yeast KlURA3 prototrophic gene (gray boxes), was linearized with EcoR I. This plasmid also contains a HIS3 gene 
and can be selected for histidine autotrophy (Additional file 1: Figure $\mathrm{S} 1$ ).

The pCORE3 plasmid and the 5' kanMX amplified product were then ligated to form pCORE6 (Additional file 1: Figures S1 and S4). In these constructions, the I-Scel restriction site is maintained in a truncated form (GATAACAGGGTAAT) (white bar) because leaky expression of the I-SceI endonuclease (if the plasmid is propagated in Escherichia coli), would result in cleavage of the pCORE3 and pCORE6 plasmids at the I-SceI site. Therefore, four additional nucleotide sequences (tagg) must be added to restore the complete 18 bp I-SceI site during amplification of the CORE6 knock-out cassette (black bar) (Figure 2A,B) (see below).

\section{B. Preparation of mutagenesis cassette for the replacement of Mmc dnaA gene by Mcc dnaA gene in Mmc genome}

A modified version of the CORE cassette described previously [22] was constructed as follows. Briefly, the CORE6 cassette includes an 18 bp I-SceI binding site (black bar), followed by a GAL1 promoter, I-Scel endonuclease gene, and KlURA3 gene (gray boxes). The CORE6 contains an additional sequence, which includes the $5^{\prime}$ region of the kanamycin resistance gene component $\left(5^{\prime} \mathrm{kanM} X\right)$ [Promoter for the Translation Elongation Factor (PTEF) followed by the $5^{\prime}$ kanamycin resistance gene sequence (1 to $859 \mathrm{bp}$ )], which forms part of the split marker system (Figure 2, Additional file 1: Figures S1 and S4). The CORE6 cassette was amplified by polymerase chain reaction (PCR) from the plasmid pCORE6 (Figure 2B) using the chimeric primers, CORE6-F (GTT TTC CAC ATT TTT AAC AAGTGT TTA ACT ATA ATATTT TTG GAG $A C A$ AAT taggGATAA CAG GGT AATACG GAT TA) and CORE6-R-modWT (GTT AAT TTGTGG ATA ACT GTT AAT AAG TTA GGTTTA AAT AGCTAT TTT TAG GCC AGC CAT TAC GCT CGT) (Figure 3A, step 1). The chimeric primers contained about $51 \mathrm{bp}$ homology (italicized), upstream (CORE6-F) or downstream (CORE6R-modWT) to the target site on the $M m c$ genome (Additional file 1: Figure S3).

In addition, a second cassette called the knock-in module carrying the $3^{\prime}$ kanMX gene component (3' kanamycin resistance gene sequence (610 to $1357 \mathrm{bp}$ ) along with the terminator TEF), a repeat sequence (51 bp homology to the upstream target site of the modified CORE6 knockout cassette), and the replacement orthologous Mcc dnaA gene was constructed in a two-step process (Figure 3A, step 2). Two overlapping PCR amplicons were produced in the first step and assembled in a second step as described below (Figures $2 \mathrm{C}$ and $3 \mathrm{~A}$ ). In the first step, the 3 'region of the $k a n M X$ gene component containing a $250 \mathrm{bp}$ overlapping region corresponding to the $5^{\prime}$ sequence of kanMX gene component in the CORE6 cassette was generated by PCR using the plasmid pFA6a-
kanMX_AJ002680 [27] as the DNA template along with chimeric primers, 3'Kanoverlap + 5' Kan-infusion-F1 (CTG ATG ATG CAT GGT TAC TCA CC) and 3'Kan-repeatinfusion-R1 (ttc atA TTT GTC TCC AAA AAT ATT ATA GTT AAA CAC TTG TTA AAA ATG TGG AAA ACC AGT ATA GCG ACC AGC ATT C). The chimeric primer, 3'Kan-repeat-infusion-R1 included a 51 bp repeat sequence (italicized), which is complementary to the upstream sequence from the target site on $M m c$ (GTT TTC CAC ATT TTT AAC AAG TGT TTA ACT ATA ATA TTT TTG GAG ACA AAT). Similarly, another PCR fragment containing the orthologous $M c c d n a A$ gene was generated using chimeric primers, repeat-MccdanA-infusion-F2 (CTA TAC TG G TTT TCC ACA TTT TTA ACA AGT GTT TAA CTA TAA TAT TTT TGG AGA CAA Ata tga acc taa acg ata ttt taa aag) and Mcc-R1-mod (GTT AAT TTGTGG ATA ACT GTT AAT AAg tTA GGTTTA AAT AGCTAT TTT TAT TAT TTT GTT AAA ATT TTATTCTTT AAA ATATCA ACA GTC), and the $M c c$ genomic DNA as template (Figures $2 \mathrm{C}$ and $3 \mathrm{~A}$ ). The chimeric primer, repeat-MccdnaA-infusion-F2 included 51 bases complementary to the primer, 3'Kan-repeatinfusion-R1 to create the overlap between the two amplicons. The chimeric primer Mcc-R1-mod included a $50 \mathrm{bp}$ homology to the downstream target site on the $M m c$ genome. In the second step, the linear knock-in module was finally assembled by a PCR-based fusion technique [28] of the two individually synthesized PCR products, the 3' kanamycin amplicon including the 51 bp repeat, and the replacement $M c c$ dnaA amplicon also carrying the $51 \mathrm{bp}$ repeat sequence (Figures $2 \mathrm{C}$ and $3 \mathrm{~A}$ ). All primers were synthesized by Integrated DNA Technologies (Coralville, IA, USA).

\section{Preparation of mutagenesis cassette for cluster deletion and complementation in the synthetic Mmc genome}

The CORE6 cassette was PCR-amplified using the plasmid pCORE6 as template, and with chimeric primers D0152/162-F (AAA ATA AAA ATT CTC TAT AAA ATA TAT TTT GTA AAC TAG AAA GGA AAA GA T AGG GAT AAC AGG GTA ATA CGG ATT AG) and D0152/ 162-R (TTT TTA TTA AAA TAT TTT AAT TAA ATT CAT TAT ATT AAA AGG ATA AAT AA G GCC AGC CAT TAC GCT CG) (Figure 4A, step 1). In order to introduce the $50 \mathrm{bp}$ repeat sequence (italicized) ( $A A A$ ATA AAA ATT CTC TAT AAA ATA TAT TTT GTA $A A C$ TAG AAA GGA AAA GA) to the knock-in module, the 3 ' $k a n M X$ gene component was amplified by two rounds of PCR. In the first round, PCR was performed for 18 cycles using the plasmid pFA6a-kanMX_AJ002680 [27] (Figure 4A, step 2) as the DNA template along with primers, 3'Kan-F (CTG ATG ATG CAT GGT TAC TC) and 3' Kan-0158-R1 (TCT AGT TTA CAA AAT ATA TTT TAT AGA GAA TTT TTA TTT TCA GTA TAG CGA CCA 
GCA TT) to generate a 788 bp amplicon. The second round of PCR was conducted for 22 cycles using the 788 bp PCR product as the DNA template along with primers, 3'Kan-F and 3' Kan-0158-R2 (TTA TTA ATT AAT AAG GAG TAA ATC TTT TCC TTT CTA GTT TAC AAA ATA TAT TTT ATA GA) to generate a 820 bp PCR product where the $50 \mathrm{bp}$ homology (underlined) to the upstream target site was incorporated right after the 3' kanMX gene component. The knock-in gene Mmc ssrA gene (679 bp) was amplified by PCR using the synthetic $M m c$ genome (Mmc Syn1) [19] as DNA template along with primers, 0158-F ( TAT ATT TTG TAA ACT AGA AAG GAA AAG ATT TAC TCC TTA TTA ATT AAT AAT AAC AA) and 0158-R (TTT TTA TTA AAA TAT TTT AAT TAA ATT CAT TAT ATT AAA AGG ATA AAT AAA CTA ATC AAT CCT AAT AAA TAC TTA G). A final knock-in module (1,527 bp) consisting of the 3' kanMX gene component, the 50 bp repeat sequence, and the $s s r A$ gene was assembled by Gibson Assembly method [29]. All primers were synthesized by Integrated DNA Technologies (Coralville, IA, USA).

\section{Transformation and PCR analysis}

Transformation of the modified CORE6 cassette or the knock-in module was performed with lithium acetate as described previously [30]. In all experiments, about $1 \mu \mathrm{g}$ of DNA construct and $25 \mu \mathrm{g}$ of salmon sperm carrier DNA (Sigma, Saint Louis, MO) were used. Transformed yeast were plated on appropriate selection media and incubated at $30^{\circ} \mathrm{C}$ for 48 hours. Based on the markers present in the DNA cassette and the mycoplasma genome, transformed yeast cells were selected on SD medium minus His (Teknova, CA), SD medium minus His and minus Ura, or YPD containing $0.2 \mathrm{mg} / \mathrm{ml}$ geneticin after a period of recovery in YPD (Figure 2).

Yeast colonies growing on selective media were restreaked and total DNA was isolated for PCR screening [31]. The correct integration of each mutagenesis cassette was verified by PCR screening using diagnostic primers located upstream and downstream of the target sites (Figures 3 and 4 and Additional file 1: Figure S3). All primers were synthesized by Integrated DNA Technologies (Coralville, IA, USA).

\section{Transplantation}

Total DNA, including the intact donor genomic DNA from yeast colonies were isolated using a CHEF Mammalian Genomic DNA Plug Kit as per the manufacturer's instructions (Bio-Rad, Hercules, CA). DNA isolated from yeast cells carrying the $M m c$ modified genome was transplanted into Mcc recipient cells with polyethylene glycol as described previously $[21,31]$. The transplanted cells were selected for tetracycline resistance (the tet $M$ gene and the $\beta$-galactosidase genes $(\operatorname{lac} Z)$ being present on the
$M m c$ chromosome). Mmc genomic DNA containing the $M c c$ dnaA gene was isolated from the transplants using the BioRobot M48 workstation (Qiagen, Valencia, CA) as per the manufacturer's instructions. The isolated $M m c$ genomic DNA from the bacteria transplants was sequenced to confirm the precise, seamless insertion of the Mcc dnaA gene (JCVI Sequencing Facility, MD).

\section{Additional file}

Additional file 1: Figure S1. Construction of pCORE6 plasmid. The
pCORE6 plasmid was constructed from the previously constructed
pCORE3 plasmid (unpublished), and the 5' region of the kanamycin
resistance gene (5' KanMX gene component) along with its promoter,
PTEF, which was amplified from the previously described pFA6a-KanMX
plasmid. The pCORE3 plasmid includes a 14 bp incomplete I-Scel
binding site (white bar), a Gal1 promoter, an I-Scel restriction enzyme gene,
and yeast KIURA3 prototrophic gene (gray boxes). The plasmid can
be selected for HIS3 prototrophy. The pCORE6 also contains a 14 bp
incomplete I-Scel site instead of the 18 bp complete sequence for stability
reasons, and an additional 4 bp (TAGG) must be added on during PCR for
generation of the complete CORE6 knock-out cassette. Figure S2. Genes
in the two non-essential gene clusters (NEGCs) separated by the
Tn5-defined essential gene, ssrA in the Mmc synthetic genome (Mmc Syn1).
Genes 0152 - 0157 belong to the first NEGC, while genes $0159-0162$ belong
to the second NEGC. Gene 0158 is the essential ssrA gene that is present
between the two NEGCs. Figure S3. Diagnostic primers to confirm
the correct insertion of the CORE6 knock-out cassette and knock-in
cassette by TREC-IN in the Mmc genome. Diagnostic primers to assess for
the correct junctions and precise insertion of the replaced Mcc orthologous
dnaA gene, and the essential SsrA gene in the Mmc genome are listed.
Figure S4. pCORE6 sequence. The CORE6 knock-out cassette (GenBank
accession number KP282615) is color-coded as follows: the 14 bp
incomplete I-Scel binding site (red), Gal1 promoter (dark green), I-Scel
endonuclease (orange), KIURA3 gene along with its promoter and
terminator (blue), and the promoter for the translation elongation factor
(PTEF) (yellow) followed by the 5 ' region of the kanamycin resistance gene
(purple).

\section{Abbreviations}

ARS: Autonomously replicating sequence; DSB: Double strand break; Gal: Galactose promoter; His: Histidine; KanMX: Kanamycin resistance gene module; lacZ: ß-galactosidase genes; Mb: Mega base; Mcc: Mycoplasma capricolum subspecies capricolum; Mmc: Mycoplasma mycoides subspecies capri; Mmc Syn 1: Synthetic Mmc genome; Mmm: Mycoplasma mycoides subspecies mycoides; NEGC: Non-essential gene clusters; NHEJ: Non-homologous end Joining; PCR: Polymerase chain reaction; PTEF: Promoter for the translation elongation factor; SD: Synthetic minimal medium containing Dextrose; TEF: Terminator; TetM: Tetracycline resistance; TREC: Tandem repeat coupled with Endonuclease cleavage; TREC-IN: TREC-assisted gene knock-in; Ura: Uracil; YCP: Yeast Centromeric Plasmid; YPD: Rich medium containing glucose; YPG: Rich medium containing Galactose.

\section{Competing interests}

The authors declare that they have no competing interests.

\section{Authors' contributions}

SC performed the experiments related to the replacement of the McC orthologous dnaA gene in the Mmc genome and wrote the manuscript. VN helped with the design of the TREC-IN method and carried out preliminary experiments. TS-S initially performed the experiments. LM performed the experiments related to the application of TREC-IN for genome reduction in the $M m c$ genome. CW performed the replacement experiments. $C L$, $\mathrm{J}$, and SV participated in the design of the study and helped to draft the manuscript. R-YC conceived and designed the TREC-IN method and helped to draft the manuscript. All authors read and approved the final manuscript. 


\section{Acknowledgements}

We thank Eva Albalghiti for her contribution towards screening of yeast colonies, and Nacyra Asad-Garcia for the transplantation work. We also thank Dr. Alain Blanchard and Dr. John Glass for useful discussion and comments on the paper. Additional support for Dr. Joerg Jores was received from the CGIAR research program on Livestockand Fish.

\section{Funding}

This work was supported in part by the National Science Foundation [grant number IOS-1110151 (to S.V., C.L., and J.J.)], DARPA Contract \# HR0011-12-C-0063, and Synthetic Genomics, Inc. Funding for open access charge: Synthetic Genomics, Inc.

\section{Author details}

${ }^{1}$ The J. Craig Venter Institute, 9704 Medical Center Drive, Rockville 20850, MD, USA. ${ }^{2}$ INRA, UMR 1332 de Biologie du Fruit et Pathologie, F-33140, Villenave d'Ornon Bordeaux, France. ${ }^{3}$ University Bordeaux, UMR 1332 de Biologie du Fruit et Pathologie, F-33140, Villenave d'Ornon Bordeaux, France. ${ }^{4}$ International Livestock Research Institute (ILRI), Old Naivasha Road, PO Box 30709, 00100 Nairobi, Kenya.

Received: 18 October 2014 Accepted: 12 December 2014 Published: 24 December 2014

\section{References}

1. Dybvig K: Mycoplasmal genetics. Annu Rev Microbiol 1990, 44:81-104.

2. Razin S, Yogev D, Naot Y: Molecular biology and pathogenicity of mycoplasmas. Microbiol Mol Biol Rev 1998, 62(4):1094-1156.

3. Halbedel S, Stulke J: Tools for the genetic analysis of Mycoplasma. Int J Med Microbiol 2007, 297(1):37-44.

4. Cordova CM, Lartigue C, Sirand-Pugnet P, Renaudin J, Cunha RA, Blanchard A: Identification of the origin of replication of the Mycoplasma pulmonis chromosome and its use in oriC replicative plasmids. J Bacterio/ 2002, 184(19):5426-5435.

5. Lartigue C, Blanchard A, Renaudin J, Thiaucourt F, Sirand-Pugnet P: Host specificity of mollicutes oriC plasmids: functional analysis of replication origin. Nucleic Acids Res 2003, 31(22):6610-6618.

6. Renaudin J, Marais A, Verdin E, Duret S, Foissac X, Laigret F, Bove JM: Integrative and free Spiroplasma citri oriC plasmids: expression of the Spiroplasma phoeniceum spiralin in Spiroplasma citri. J Bacteriol 1995, 177(10):2870-2877.

7. Janis C, Lartigue C, Frey J, Wroblewski H, Thiaucourt F, Blanchard A, Sirand-Pugnet $P$ : Versatile use of oriC plasmids for functional genomics of Mycoplasma capricolum subsp. capricolum. Appl Environ Microbiol 2005, 71(6):2888-2893.

8. Lee SW, Browning GF, Markham PF: Development of a replicable oriC plasmid for Mycoplasma gallisepticum and Mycoplasma imitans, and gene disruption through homologous recombination in $\mathrm{M}$. gallisepticum. Microbiology 2008, 154(Pt 9):2571-2580.

9. Janis C, Bischof D, Gourgues G, Frey J, Blanchard A, Sirand-Pugnet P: Unmarked insertional mutagenesis in the bovine pathogen Mycoplasma mycoides subsp. mycoides SC: characterization of a IppQ mutant. Microbiology 2008, 154(Pt 8):2427-2436.

10. Hutchison CA, Peterson SN, Gill SR, Cline RT, White O, Fraser CM, Smith HO, Venter JC: Global transposon mutagenesis and a minimal Mycoplasma genome. Science 1999, 286(5447):2165-2169.

11. Glass Jl, Assad-Garcia N, Alperovich N, Yooseph S, Lewis MR, Maruf M, Hutchison CA 3rd, Smith HO, Venter JC: Essential genes of a minimal bacterium. Proc Natl Acad Sci U S A 2006, 103(2):425-430.

12. Voelker LL, Dybvig K: Transposon mutagenesis. Methods Mol Biol 1998, 104:235-238.

13. Burgos R, Pich $O Q$, Querol E, Pinol J: Deletion of the Mycoplasma genitalium MG_217 gene modifies cell gliding behaviour by altering terminal organelle curvature. Mol Microbiol 2008, 69(4):1029-1040.

14. Dhandayuthapani S, Blaylock MW, Bebear CM, Rasmussen WG, Baseman JB: Peptide methionine sulfoxide reductase (MsrA) is a virulence determinant in Mycoplasma genitalium. J Bacteriol 2001, 183(19):5645-5650.

15. Dhandayuthapani S, Rasmussen WG, Baseman JB: Disruption of gene mg218 of Mycoplasma genitalium through homologous recombination leads to an adherence-deficient phenotype. Proc Natl Acad Sci U S A 1999, 96(9):5227-5232
16. Allam AB, Reyes L, Assad-Garcia N, Glass Jl, Brown MB: Enhancement of targeted homologous recombination in Mycoplasma mycoides subsp. capri by inclusion of heterologous recA. Appl Environ Microbiol 2010, 76(20):6951-6954.

17. Benders GA, Noskov VN, Denisova EA, Lartigue C, Gibson DG, Assad-Garcia N, Chuang RY, Carrera W, Moodie M, Algire MA, Phan Q, Alperovich N, Vashee S, Merryman C, Venter JC, Smith HO, Glass JI, Hutchison CA 3rd: Cloning whole bacterial genomes in yeast. Nucleic Acids Res 2010, 38(8):2558-2569.

18. Gibson DG, Benders GA, Andrews-Pfannkoch C, Denisova EA, Baden-Tillson H, Zaveri J, Stockwell TB, Brownley A, Thomas DW, Algire MA, Merryman C, Young L, Noskov VN, Glass JI, Venter JC, Hutchison CA 3rd, Smith HO: Complete chemical synthesis, assembly, and cloning of a Mycoplasma genitalium genome. Science 2008, 319(5867):1215-1220.

19. Gibson DG, Glass Jl, Lartigue C, Noskov VN, Chuang RY, Algire MA, Benders GA, Montague MG, Ma L, Moodie MM, Merryman C, Vashee S, Krishnakumar R, Assad-Garcia N, Andrews-Pfannkoch C, Denisova EA, Young L, Qi ZQ, Segall-Shapiro TH, Calvey CH, Parmar PP, Hutchison CA 3rd, Smith HO, Venter JC: Creation of a bacterial cell controlled by a chemically synthesized genome. Science 2010, 329(5987):52-56.

20. Lartigue C, Glass Jl, Alperovich N, Pieper R, Parmar PP, Hutchison CA 3rd, Smith $\mathrm{HO}$, Venter JC: Genome transplantation in bacteria: changing one species to another. Science 2007, 317(5838):632-638.

21. Lartigue C, Vashee S, Algire MA, Chuang RY, Benders GA, Ma L, Noskov VN, Denisova EA, Gibson DG, Assad-Garcia N, Alperovich N, Thomas DW, Merryman C, Hutchison CA 3rd, Smith HO, Venter JC, Glass J: Creating bacterial strains from genomes that have been cloned and engineered in yeast. Science 2009, 325(5948):1693-1696.

22. Noskov VN, Segall-Shapiro TH, Chuang RY: Tandem repeat coupled with endonuclease cleavage (TREC): a seamless modification tool for genome engineering in yeast. Nucleic Acids Res 2010, 38(8):2570-2576.

23. Akada R, Hirosawa I, Kawahata M, Hoshida H, Nishizawa Y: Sets of integrating plasmids and gene disruption cassettes containing improved counter-selection markers designed for repeated use in budding yeast. Yeast 2002, 19(5):393-402.

24. Daley JM, Palmbos PL, Wu D, Wilson TE: Nonhomologous end joining in yeast. Annu Rev Genet 2005, 39:431-451.

25. Jores J, Mariner JC, Naessens J: Development of an improved vaccine for contagious bovine pleuropneumonia: an African perspective on challenges and proposed actions. Vet Res 2013, 44:122.

26. Boeke JD, LaCroute F, Fink GR: A positive selection for mutants lacking orotidine-5'-phosphate decarboxylase activity in yeast: 5-fluoro-orotic acid resistance. Mol Endocrinol 1984, 197(2):345-346.

27. Wach A, Brachat A, Pohlmann R, Philippsen P: New heterologous modules for classical or PCR-based gene disruptions in Saccharomyces cerevisiae. Yeast 1994, 10(13):1793-1808.

28. Shevchuk NA, Bryksin AV, Nusinovich YA, Cabello FC, Sutherland M, Ladisch S: Construction of long DNA molecules using long PCR-based fusion of several fragments simultaneously. Nucleic Acids Res 2004, 32(2):e19.

29. Gibson DG, Young L, Chuang RY, Venter JC, Hutchison CA 3rd, Smith HO: Enzymatic assembly of DNA molecules up to several hundred kilobases. Nat Methods 2009, 6(5):343-345.

30. Gietz D, St Jean A, Woods RA, Schiestl RH: Improved method for high efficiency transformation of intact yeast cells. Nucleic Acids Res 1992 20(6):1425.

31. Noskov V, Kouprina N, Leem SH, Koriabine M, Barrett JC, Larionov V: A genetic system for direct selection of gene-positive clones during recombinational cloning in yeast. Nucleic Acids Res 2002, 30(2):E8.

doi:10.1186/1471-2164-15-1180

Cite this article as: Chandran et al:: TREC-IN: gene knock-in genetic tool for genomes cloned in yeast. BMC Genomics 2014 15:1180. 